\title{
Neurogenic bowel dysfunction in patients with multiple sclerosis: prevalence, impact, and management strategies
}

This article was published in the following Dove Press journal: Degenerative Neurological and Neuromuscular Disease

\section{Giuseppe Preziosi Ayeshah Gordon-Dixon Anton Emmanuel}

Gastro-Intestinal Physiology Unit, University College London Hospital, London, UK
Correspondence: Giuseppe Preziosi Gastro-Intestinal Physiology Unit, University College London Hospital, 235 Euston Road, Fitzrovia, London, NWI 2BU, UK

Tel +440203447 7475

Email g.preziosi@nhs.net

\begin{abstract}
Bowel dysfunction in patients with multiple sclerosis (MS) is highly prevalent. Constipation and fecal incontinence can coexist and alternate, impacting on the patient's quality of life and social interactions, as well as burdening the caregivers. The cost for the health care providers is also significant, with increased number of hospital admissions, treatment-related costs, and hospital appointments. The origin is multifactorial, and includes alteration of neurological pathways, polypharmacy, behavioral elements, and ability to access the toilet. Every patient with MS should be sensitively questioned about bowel function, and red flag symptoms should prompt adequate investigations. Manipulation of life style factors and establishment of a bowel regime should be attempted in the first place, and if this fails, other measures such as biofeedback and transanal irrigation should be included. A stoma can improve quality of life, and is not necessarily a last-ditch option. Antegrade colonic enemas can also be an effective option, whilst neuromodulation has not proved its role yet. Effective treatment of bowel dysfunction improves quality of life, reduces incidence of urinary tract infection, and reduces health care costs.
\end{abstract}

Keywords: neurogenic bowel dysfunction, multiple sclerosis, constipation, fecal incontinence

\section{Introduction}

It is estimated that multiple sclerosis (MS) affects around 2.3 million patients worldwide and is the commonest non-traumatic cause of disability in young adults. ${ }^{1}$ The underlying pathological mechanism is autoimmune, causing demyelination within the central nervous system, which leads to the formation of plaques in the white matter and dysfunction of the neurological pathways affected. Clinically, this results in a multitude of neurological symptoms and eventually severe disability. MS is classified according to how the symptoms present and progress. The most common type is defined as relapsing-remitting, which accounts for up to $80 \%$ of cases, whilst progressive disease is classified as either secondary or primary progressive. Commonly symptoms fluctuate and/or progress, and eventually accumulate in relation to the axonal loss and atrophy within the brain and spinal cord. ${ }^{2}$ Disability is commonly quantified with the Expanded Disability Status Scale (EDSS), ${ }^{3}$ which correlates well with the degree of spinal cord atrophy. ${ }^{4}$ The precise etiology for this disease process is still unknown, and whilst the quest for a definitive treatment is ongoing, in the absence of a cure, improvement in symptoms management is imperative.

We will discuss in this review the pathophysiology of MS-related bowel dysfunction, reviewing the essential anatomy and physiology of defecation, the psychosocial and financial impact, and management strategies. 


\section{Methods}

The authors conducted a search on PubMed/MeSH and Medline databases, using the terms "multiple sclerosis" in combination with "bowel symptoms", "constipation", "fecal incontinence", and "neurogenic bowel dysfunction", which was also searched independently. We then perused the bibliography to extract any further study including published abstracts. Given the paucity of studies, particularly on treatment, a systematic review was not possible, and this is therefore a narrative review, which includes all studies on the pathophysiology of MS-related bowel dysfunction and its treatment. With regards to symptom management strategies, any recommendation is based on the 2014 Cochrane review on the management of neurogenic bowel dysfunction ${ }^{5}$ (NBD) and on expert consensus. ${ }^{6}$

\section{NBD in patients with MS}

NBD is a prevalent condition amongst patients with MS, affecting overall between $39 \%$ and $73 \%$ of sufferers depending on the population studied. The presence of bowel symptoms appears to be correlated to the EDSS and disease duration, but not with the type of MS. ${ }^{7-11}$ Nevertheless, patients with low disability and a short illness can have bowel symptoms and, in fact, severe constipation has been reported as the first presenting symptom of MS. ${ }^{12}$

The definition of constipation is commonly subject to individual perception, and generally refers to infrequent bowel action and difficulty in releasing stools. A constellation of symptoms might be present, such as tiredness, bloating, and abdominal pain. Whilst it remains a symptom and not a diagnosis, constipation is defined by the Rome criteria $^{13}$ (Box 1), which should have been fulfilled for the last 3 months, with symptom onset at least 6 months prior to diagnosis of functional constipation. ${ }^{14}$

Fecal incontinence (FI) is the involuntary loss of stools or flatus for at least 1 month. ${ }^{15}$ This can occur without the patient being aware of it (passive FI), or it can be accompanied by a

Box I Rome criteria to define constipation, by the presence of two or more of these symptoms in at least $25 \%$ of the defecations

- Straining during defecation

- Hard or lumpy stools

- Sensation of incomplete evacuation

- Sensation of anorectal blockage or obstruction

- Use of manual maneuvers to facilitate evacuation (includes digitation to the perineum)

- Three or less defecations per week

- Rarely has loose stools without the use of laxatives

Note: Data from Drossman. ${ }^{13}$ variable degree of urgency (urge FI). Like for constipation, it is clinically very important to qualify accompanying stools consistency.

Constipation is present in half of the patients with MS, and FI affects about $50 \%$ of patients at some point and is an ongoing symptom in about $25 \%$ of cases. ${ }^{8}$ Commonly, constipation and incontinence are coexistent and alternating. Considering that FI affects $2 \%$ of the population ${ }^{16}$ and constipation $2 \%-20 \%,{ }^{17}$ it is clear that bowel dysfunction is far more prevalent in MS patients than the general population.

\section{Psychosocial and physical impact}

Patients with MS ranked bowel problems as the third most bothersome symptom after fatigue and issues with mobility. ${ }^{18}$ This is not surprising given the embarrassing nature of bowel symptoms, and the need to plan activities of daily living around bowel care which will affect a persons' approach to social interactions. ${ }^{19}$ Consequentially, bowel symptoms are considered the primary cause of inability to work, after spasticity and incoordination. ${ }^{20}$ These factors, amongst the other associated physical challenges, may lead to significant psychological and emotional insult. ${ }^{21}$

The experience of constipation will vary amongst patients with MS and the variables will be factors related to the extent of difficulty to evacuate, such as how long does the person need to spend on the toilet, if they need perineal or rectal stimulation, and if they regularly have problems with impaction requiring manual evacuation. In a survey, it was found that a third of MS sufferers spend at least 30 minutes for their bowel toileting, ${ }^{19}$ but in clinical experience it is not uncommon, particularly (but not necessarily) in the most disabled patients, that toileting takes most of the day in designated days of the week. In fact, some patients might hardly ever leave the house because of repeated attempts to open their bowels, or for the fear of FI.

It only takes one occurrence of an episode of FI, no matter the initial reason, to propagate enough anxiety which may lead to isolation and self-imposed restrictions in order to avoid the risk of what an individual will see as embarrassment, shame, and humiliation if it were to happen.

Anxiety disorders and depression are mental states that become a part of the presenting complaint once NBD starts to manifest, and they are improved along with successful treatment. ${ }^{22}$ Although multiple explanations can justify what could possibly be a "chicken-or-egg" correlation, ${ }^{23}$ it goes to show a significant psychological component associated with bowel dysfunction in MS patients as in the normal population. ${ }^{24}$ 
As such, these feelings may also contribute to patients presenting late, having persevered, endured, and managed the symptoms with gradual adaptation even as symptoms have progressed over time. Caregivers may inadvertently enable this behavior as they also adapt to the effects of NBD in order to be supportive and to avoid causing them distress, especially when the caregiver is a family member. ${ }^{18}$ The caregivers themselves will be affected by the increased need for support, if the patients with MS is losing their independence, confidence, and self-esteem as the level of care increases. Ultimately, these patients are likely to become socially withdrawn, including reluctance to go and connect with friends or family, take holidays, and may become housebound as with each venture there is the need to plan access to toileting facilities..$^{19,21}$

Other concurrent symptoms, such as hemorrhoids, abdominal pain, fecal impaction, rectal bleeding, rectal prolapse, anal fissure, abdominal bloating, nausea, autonomic dysreflexia, and prolonged evacuation, can complicate NBD contributing to impaired quality of $\mathrm{life}^{25-30}$ and aggravate the physical impact of NBD. Food restrictions, in an attempt to avoid incontinence, can lead to weight loss. On the other hand, the inability to work can lead to a nutritionally poor diet.

\section{Economic impact}

The cost of standard bowel care for NBD-related FI is just under $£ 10,000$ per patient. Symptomatic patients are more likely to seek medical attention from the general practitioner, medical specialists, and dieticians; they are twice as likely to require emergency hospital admission which may be related to fecal impaction, megacolon, and volvulus, ${ }^{31}$ and the estimated cost of a typical hospital admission for constipation in a patient with MS is estimated at $£ 1,729 .{ }^{32}$ Some patients will go on to require elective or emergency surgery followed by inpatient stay to manage NBD.

Effective treatment of NBD is indeed associated with a reduction in need for hospitalization, physician visits, number of urinary tract infections, and need for surgery, ${ }^{33}$ with expected cost reductions. ${ }^{34}$ Patients with severe symptoms might be less likely to be employed, ${ }^{20}$ and although no data exist to quantify the societal financial impact, it is likely to be significant.

\section{Mechanism of defecation}

Normal bowel function is multifactorial and relies not only on the anatomical integrity of the bowel and its innervation, but also on hormonal factors and adequate nutrition, which in turn will affect consistency of intra-luminal content. Furthermore, the ability to access the toilet and a behavioral component are essential in maintaining normal bowel function.

Schematically, once the stools have reached the large bowel, effective colonic transit will allow manipulation of stool consistency, reabsorbing water and secreting mucous to form the stools. Mass colonic contractions will deliver the stools in the rectum, which acts as a reservoir. Essential physiological attributes for effective rectal function are good compliance and ability to sense its luminal content. The rectoanal inhibitory reflex (RAIR, Figure 1) will ensure that rectal content is identified at somatic level and released if and when it is perceived to be a socially acceptable time.

The areas of the brain thought to be involved in gut function include the cingulate cortex, insula, thalamus, somatosensory cortex, and prefrontal cortex. ${ }^{35}$

The extrinsic innervation pathway to the bowel involves somatic and visceral sensorial fibers. Somatic afferents are via the pudendal nerve which innervates the external anal sphincter and provides anal sensation. Parasympathetic nerves of the sacral plexus S2-S4 are responsible for rectal sensation. Proximal colonic innervation is from the vagus nerve for the parasympathetic stimulating and sensorial component, whilst the thoracic spinal cord (T5-L2) supplies inhibitory sympathetic fiber to the colon and rectum. The internal anal sphincter has tonic sympathetic activity which helps to maintain the resting tone of the anal canal. The voluntary striated muscle fibers of the external sphincter are also supplied by the pudendal nerve, from the Onuf's nucleus, via the ventral horn of the spinal cord. ${ }^{36}$

The intrinsic pathway is called the enteric nervous system (ENS) which has two components: the myenteric plexus, which controls the smooth muscles of the gut, and the submucosal plexus, which regulates the secreto-motor and sensory components of gut function. The ENS works to coordinate peristalsis, secretion, and absorption of luminal content within the gut. ${ }^{37}$

The spinal cord conducts signals from gut to brain and vice versa within the dorsal column and spinothalamic tracts. The act of defecation is the result of a coordinated reflex activity at spinal cord level, which is modulated and influenced by the cortex.

The precise neuropathological mechanism underlying NBD in MS results is not defined. However, the disease process can affect these pathways directly and at multiple levels.

At cortical level, demyelination within the frontal lobe may affect one's voluntary control over defecation. ${ }^{38}$

The role of the spinal cord in maintaining normal bowel function is evident, as NBD is almost invariably present in 


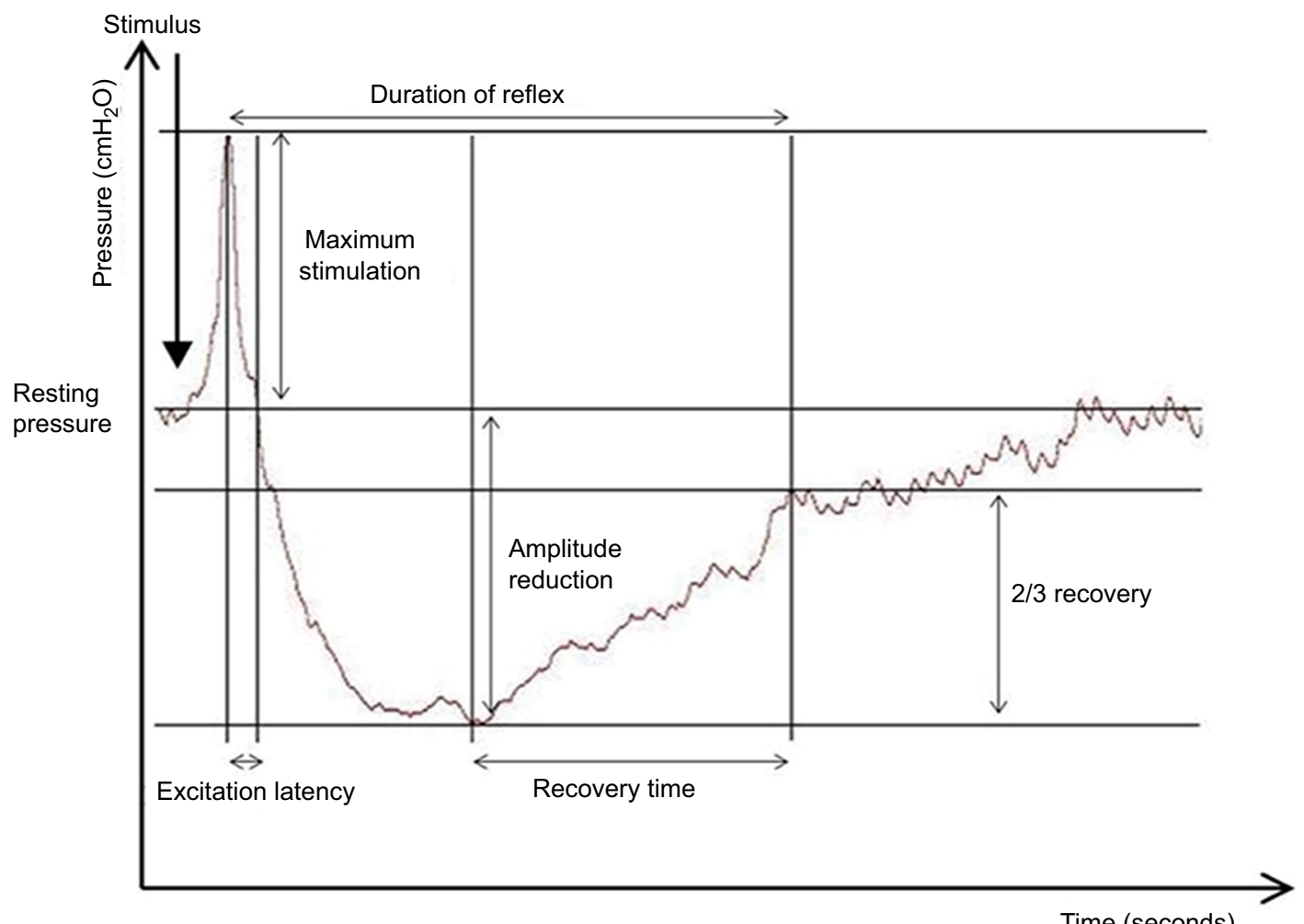

Time (seconds)

Figure I Rectoanal inhibitory reflex (RAIR).

Notes: Excitation peak: initial increase in the resting pressure is associated with sudden rectal distension. Excitation latency: duration from the point of excitation peak back to the baseline pressure. Point of maximum relaxation: lowest point of resting pressure secondary to reflex internal anal sphincter relaxation. Recovery time: the duration between maximum relaxation and the point at which the resting pressure recovers to two-thirds its baseline value. Total reflex duration: calculated as the duration from the point of the excitation peak to the point where two-thirds recovery is observed. Figure reproduced from Thiruppathy K, Roy A, Preziosi G, Pannicker J, Emmanuel A. Morphological abnormalities of the recto-anal inhibitory reflex reflects symptom pattern in neurogenic bowel. Dig Dis Sci. 2012;57(7): 1908-1914. Copyright 2012 Thiruppathy et al. ${ }^{47}$

patients with a spinal cord injury (SCI) ${ }^{39}$ In patients with MS, it has been shown that there are delays in the motor pathways to the anal sphincter, ${ }^{40,41}$ as well as delay in generated evoked potentials within the spinal cord. ${ }^{42}$

Most studies have focused on measuring alterations on the end organ, particularly the anorectal unit. Anal sphincter has been observed to be weak, and anorectal sensation has been shown to be reduced in some patients with bowel symptoms. ${ }^{10,11}$ Whilst these findings are certainly significant, no conclusion can be made on the underlying pathophysiology.

Rectal compliance is a measure of the ability of the rectum to distend, and its alterations are well documented cause of both $\mathrm{FI}^{43}$ and constipation. ${ }^{44}$ In patients with NBD, the tone and function of the smooth muscle of the rectal wall can be altered resulting in impaired compliance, as shown in patients with a SCI. ${ }^{45}$ These findings suggest a primary role of the spinal cord in maintaining rectal compliance. Crucial evidence in demonstrating the role of the spinal cord in determining bowel symptoms in patients with MS came from a study which showed that rectal compliance was altered in a predictable manner, depending on the clinical degree of spinal cord disease, and that patients with higher EDSS had a rectal compliance similar to that of patient with a supraconal SCI. On the contrary, patients with low disability had rectal compliance similar to that of normal controls. ${ }^{46,47}$

Rectal sensation and the RAIR both depend on rectal distension and are thus related to rectal compliance. Alterations of these three elements have been studied separately, but might in fact act in synergy. ${ }^{44}$

Pelvic floor dyssynergia occurs when abdominal and rectal wall contraction are not coordinated with pelvic floor relaxation, resulting in the inability to evacuate the rectum, and is commonly observed in patients with functional constipation. ${ }^{48}$ This phenomenon, also called anismus, has been well documented in MS patients. ${ }^{49,50}$ In non-neurological patients, pelvic floor dyssynergia is behavioral in nature. The same might apply to MS patients, but it could also be a pelvic floor manifestation of spasticity and incoordination, and parallel to bladder detrusor dyssynergia. ${ }^{51}$

Improvement of pelvic floor dyssynergia can normalize colonic transit in idiopathic constipation, ${ }^{52}$ and this goes to show how the function of the anorectal unit is clearly integrated with large bowel motility. With regards to the activity of the more proximal large bowel, slow colonic transit is a 
feature which has been repeatedly observed. It is possible that this is secondary to anismus, but could be secondary to unopposed sympathetic inhibitory outflow due to spinal cord plaques $^{53,54}$ or secondary to a dampened gastrocolic reflex. ${ }^{55}$
In the same study of colonic function, uncontrolled peristalsis was observed in patients with MS and diarrhea. ${ }^{55}$

A schematic view of how all these alterations can interplay to cause NBD is summarized in Figures 2 and 3.

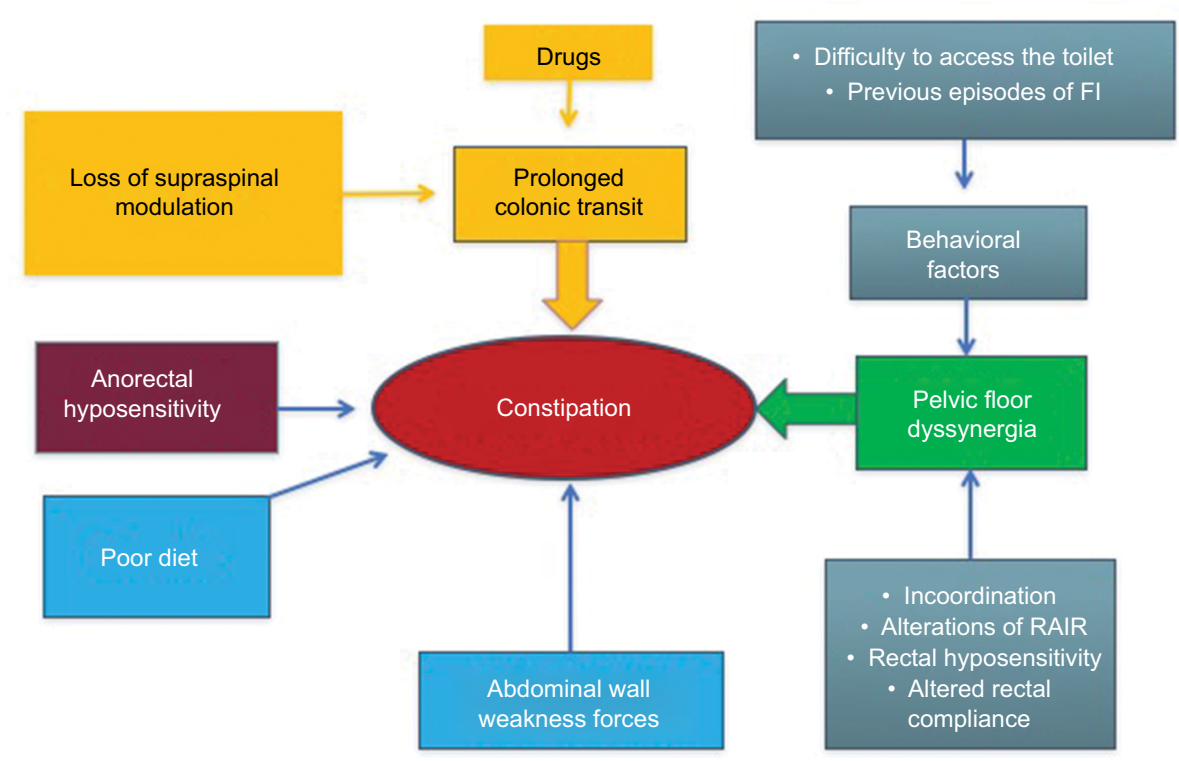

Figure 2 Diagram showing the multifactorial origin of constipation.

Notes: Adapted from Preziosi G. Pathophysiology of Bowel Dysfunction in Multiple Sclerosis and the potential for targeted treatment [Doctoral thesis]. London, UK: University College London; 2014. Copyright 2014 Preziosi. ${ }^{85}$

Abbreviations: FI, fecal incontinence; RAIR, rectoanal inhibitory reflex.

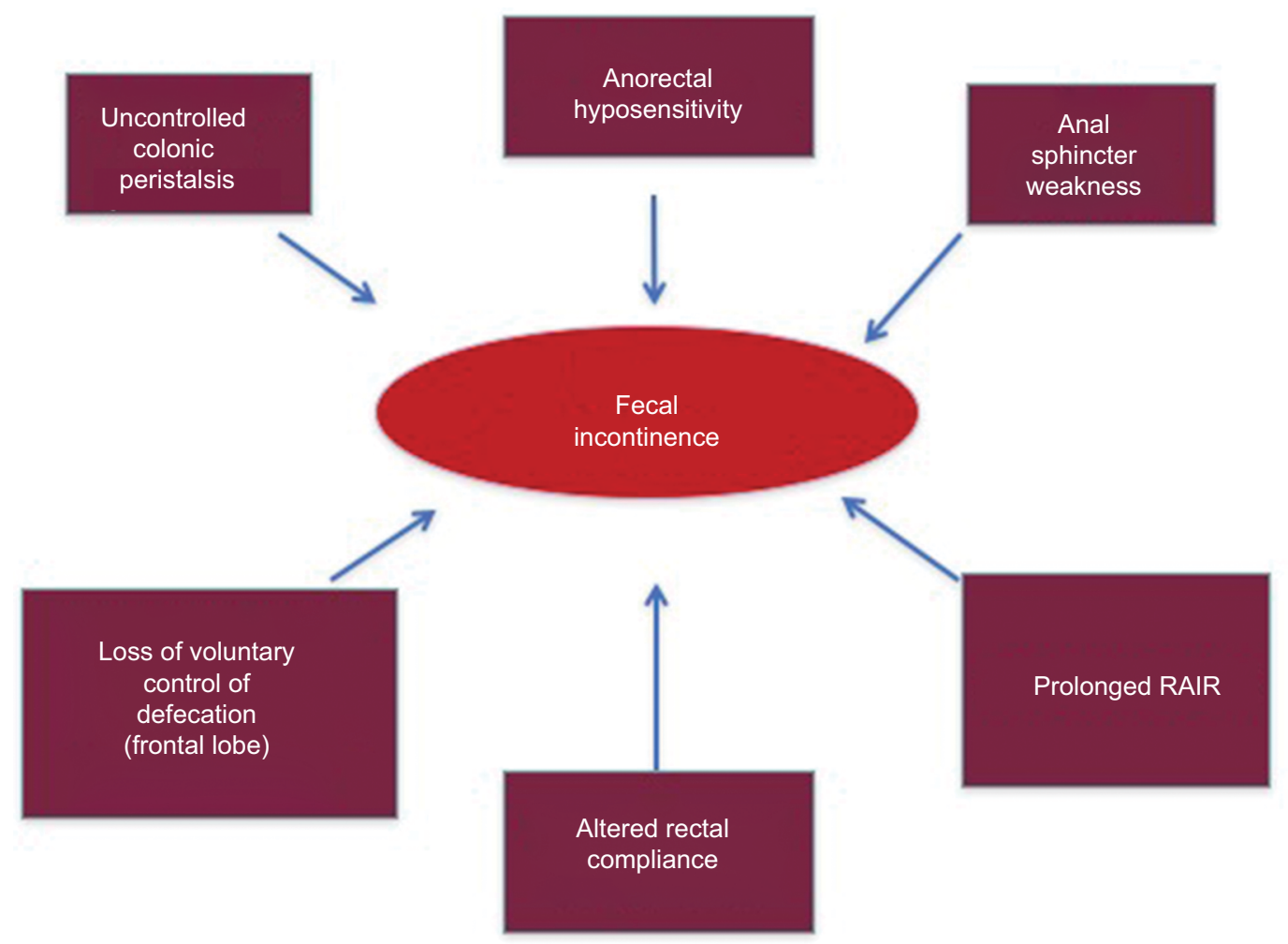

Figure 3 Diagram showing the multifactorial origin of fecal incontinence.

Notes: Adapted from Preziosi G. Pathophysiology of Bowel Dysfunction in Multiple Sclerosis and the potential for targeted treatment [Doctoral thesis]. London, UK: University College London; 2014. Copyright 2014 Preziosi. $^{85}$

Abbreviation: RAIR, rectoanal inhibitory reflex. 


\section{Treatment}

\section{Symptoms assessments}

It is well documented that FI is considered a taboo topic by both patients and physicians, as observed in the general population. ${ }^{56-58}$ Furthermore, in patients with MS and their caregivers there might be a passive acceptance of these symptoms as normal. To compound this, bowel symptoms can be perceived to be less relevant within the bigger picture of their disease. ${ }^{21}$ Over the last few years, there has been increased awareness of the need to enquire about bowel function in patients with MS. ${ }^{59} \mathrm{It}$ is worth remembering that bowel symptoms can be present regardless of disease duration, type, or level of disability.

We have described the potential neuropathological mechanisms underlying bowel symptoms, but it should be always in the mind of the assessing physician or nurse its multifactorial nature and the need for a holistic assessment.

Any "red flag" symptoms, such as the presence of blood in the stools, a change in bowel habit with no apparent cause (disease relapse, change in lifestyle or medications), or unexplained weight loss should prompt endoscopic evaluation and/or cross-sectional imaging. Bowel function before MS diagnosis needs to be explored, including any history of an eating disorder. An assessment of diet and nutritional status includes evaluation of fluid intake, which is often restricted in order to prevent bladder symptoms.

Coexistent symptoms of foregut dysmotility should also be sought, asking for symptoms of early satiety and abdominal distension.

Comorbidities should be carefully evaluated, including diabetes and a history of obstetric injuries. Given the common neurological pathways of pelvic organs, urogenital dysfunction needs to be adequately assessed. It should also be noted that a higher incidence of inflammatory bowel disease is observed in patients with MS. ${ }^{60}$

A detailed drug history could reveal the use of constipating agents, particularly anti-muscarinic drugs and drugs used for spasticity such as baclofen. Polypharmacy in general can be a cause of constipation, whilst metformin, statins, and antibiotics might be responsible for loose stools.

Patients with MS might have a normal and preserved urge to defecate, but this can be reduced or completely lost and/or associated with a sudden urgency. In some patients, the need to open the bowels can present as abdominal pain and occasionally as the worsening of symptoms of spasticity. Evaluation of frequency of a bowel action and time spent in the toilet are important as well as stool consistency, and the Bristol stool chart can be useful. Behavioral aspects need questioning, including multiple attempts to empty the bowel and toilet awareness, which is a need for the patient who is constantly mapping the presence of toilets, for example, on the route to work. Patients might complain of difficulty with wiping clean, which is a sign of incomplete rectal emptying.

Symptoms of FI, including urgency, are often undisclosed and should be sensitively explored.

Bowel symptoms in MS patients cover a spectrum, which includes at one end the extreme symptoms of obstinate constipation and at the other of complete passive FI. In many patients, both constipation and incontinence can be present, and in fact correlated, as reduced rectal sensitivity will not provide any awareness of rectal fullness, with subsequent sudden onset of urgency resulting in FI.

With regards to physical examination, digital rectal examination (DRE) is crucial. It could reveal any complication of bowel dysfunction, such as hemorrhoids or fissures, as well as allowing to assess for the presence of hard stools or fecal impaction which are signs not only of constipation but is also causative of overflow diarrhea. DRE also allows a crude assessment of anal sensation, as well as anal tone and squeeze pressure.

Specialist tests are indicated in patients with severe or persistent symptoms. They include tests of anorectal physiology, which will give an accurate measure of somatovisceral anorectal sensation and anal sphincter pressures, colonic transit time studies, and endoanal ultrasound assessing integrity of the anal sphincters. MRI proctography is occasionally used to evaluate the presence of enteroceles or pelvic floor dyssynergia.

Accurate quantification of symptoms includes the use of a bowel diary for 2 weeks, which will avoid recollection bias and give both the physician and patient a realistic evaluation of frequency of symptoms. The NBD questionnaire, designed for SCI patients, gives a good evaluation of symptoms and impact on quality of life. However, simple questioning on the percentage $(0 \%, 25 \%, 50 \%, 75 \%$, and $100 \%)$ by which bowel symptoms are affecting the patient's life, ${ }^{61}$ or more simply if they are absent, mild, moderate, or severe, will give a comparable good assessment of their severity. ${ }^{62}$

The Wexner Constipation and Incontinence scores give a good quantitative assessment and have been employed before with MS patients. Other questionnaires, such as the St Marks Score, is more useful in a research setting.

\section{Bowel regime}

At present, the management of bowel dysfunction in MS patients remains unsatisfactory, ${ }^{63}$ and much of the evidence 
available on the treatment of NBD comes from studies in patients with a SCI, with whom MS patients share many similarities.

The establishment of a bowel regime requires a thorough understanding of the patient's symptoms as well as his or her need for social interaction, work engagements, and level of support available both from caregivers and local institutions. Whether treatment has failed or not attempted yet, the aim is to develop a routine that works for the patient. The first step is to engage the patient and the primary caregiver in a process that might require trial and failure, with the understanding that any intervention might worsen symptoms and might not have a long-lasting effect. In fact, it is of paramount importance that both physician and patient are aware that the treatment of bowel dysfunction follows the opposite of the rule where "one size fits all". Whilst occasionally success can be quickly and easily obtained with a laxative or anti-diarrheal prescription, the patient and caregivers, as well as the physician or nurse treating patients with MS, need to understand that improvement might require time, titration of medications, and changes in lifestyle. In other words, both health care practitioners and patients need to agree on embarking in a journey, and that it unlikely that one single visit will successfully address symptoms.

Manipulation of stool consistency if necessary is very important and can be achieved by modulating water and fiber intake. The balance to strike is between having enough fiber to have soft formed stools, preventing incontinence and constipation, and at the same time avoiding bloating, which can occur with excessive fiber intake or with the use of laxatives, in the presence of pan-gut dysmotility. The presence of the latter can be only presumed but not always be established in the clinical setting, hence the importance of explaining this to the patient, so that fiber intake can be adjusted accordingly during the development of a bowel regime.

A cautious use of a constipating agent can often significantly improve FI. ${ }^{59}$ Depending on patient's engagement and ability, the titration of loperamide syrup can allow to quantify exactly the dose required to improve stools consistency without causing constipation. The patient could then move to the tablet form, if more convenient, at the established dose. Anti-diarrheal agents should be available to be used as required when traveling or for social engagements.

Excessive caffeine, alcohol, or foodstuffs containing sorbitol can also cause loose stools. ${ }^{64}$

Strategies to maximize bowel emptying will prevent fecal impaction as well as incontinence and include maximization of laxative effect where required.
Additional measures might include abdominal massage, using the heel of the palm in a right to left circular motion. ${ }^{65}$

It should be remembered that addressing constipation will often ameliorate symptoms of FI if present, and so we will look at overall available treatment modality, rather than looking at treatment of a specific symptom.

\section{Laxatives}

Scant evidence exists on the use of laxatives, mostly from studies of neurological condition or idiopathic bowel symptoms; however, clinical experience dictates that a laxative regime needs to be tailor made, as many variables affects their efficacy. So regardless of the evidence, more than one laxative might need to be attempted before satisfactory effects are obtained. Therefore, it is important to explain to the patient, potential lack of beneficial effect as well as side effects, such as bloating or diarrhea, and that any occurrence should not be considered as a failure of treatment, but rather an important step of the trial and error process. It is also important to underline that a positive effect from a laxative can be subjective, and could increase frequency of bowel action, amelioration of ancillary symptoms, or improved feeling of evacuation.

Psyllum is a bulking agent which might work for slowtransit constipation as shown in patient with Parkinson's disease (PD). ${ }^{66} \mathrm{~A}$ bulking agent might also work in patients who are wheelchair bound. ${ }^{67}$

The use of the osmotic agent lactulose should be cautious and used only in patients with very mild symptoms. In fact, given the likely presence of pan-gut dysmotility, it is likely to cause or aggravate symptoms of bloating, due to fermentation and production of methane, as well as being ineffective.

Polyethylene glycol equally acts as osmotic agent but does not rely on bacterial fermentation for its activation. The advantage is that it can be quite effective, and the dose can be adjusted within a wide range according to patient's need. In patients with $\mathrm{PD}$, it has proved to relieve constipation. ${ }^{68}$

A stimulant laxative such as bisacodyl has been shown to be very effective in patients with chronic idiopathic constipation. ${ }^{69}$

In clinical experience, these laxatives can work in MS patients when used in a stepwise approach in the order in which they have been mentioned.

The use of rectal stimulants or digitation can be employed. Bisacodyl suppositories act as rectal stimulants, having a very rapid onset of action as shown in patients with a spinal cord injury. ${ }^{70}$ 
On average, laxatives take around 8 hours to have an effect. Therefore, within a bowel regime, they are taken in the evening, so that optimal effect can be achieved in the following morning. Efficacy will be maximized by the mass contractions, typical of the morning rising, and the gastrocolic reflex, as a result of breakfast and a hot drink.

However, due to variability in gut transit time, the effects can be unpredictable. Both patient and caregiver should observe this and implement a regime accordingly.

A combination of different laxatives might be more effective, with or without the addition of a rectal suppository.

\section{Prucalopride}

Prucalopride is a highly selective 5-HT4 agonist that has been shown to be effective in patients with chronic constipation. ${ }^{71}$ The most frequently reported adverse events of prucalopride were headache, nausea, abdominal pain, and diarrhea, which were mainly reported on the first day of treatment. In nonneurogenic constipation, it increases colonic motility and thus improves colonic transit time, and can be considered as second-line treatment for constipation in patients with MS. Medaer et al conducted a small study with patients having treatment for 4 weeks and reported that patients had improvement in constipation symptoms; however, a larger trial is required to strengthen the evidence as this was a small randomized controlled trial $(\mathrm{RCT}) .^{72}$

\section{Biofeedback (BF)}

Bowel $\mathrm{BF}$, in its more conservative definition, is a technique that aims at the modification of bowel function through behavioral changes with the aid of an external device. The device will allow visualization of a function, for example, anal sphincter contraction, and feed it back to the patient who can then work on modifying it. ${ }^{73} \mathrm{BF}$ addresses pelvic floor dyssynergia, as well as sphincter weakness and the ability to identify rectal content (rectal sensitivity), whilst learning to resist urge to defecate, and can ameliorate both symptoms of constipation and FI. Currently, there is no agreement on a standardized technique, and provision of this service is very patchy.

Three studies have demonstrated its efficacy in patients with MS, not only with regards to symptoms, but also in improvement of physiological parameters such as squeeze and endurance anal sphincter pressures. ${ }^{22,74,75}$ This in turn suggests that there can be a significant behavioral component in MS-related bowel dysfunction. BF can induce measurable autonomic changes, which are mediated by the spinal cord, and recruitment of residual spinal cord function could be responsible for the observed physiological changes. ${ }^{52}$ This would be in accordance with the observation that patients with less disability appear to benefit more from BF. Whilst no strict criteria can be extrapolated from the literature, $\mathrm{BF}$ is very unlikely to work in patients who are wheelchair bound and have lost completely anorectal function. The latter can be proved with test of anorectal physiology in case of uncertainty, but clinical examination should reveal complete loss of sensation, lack of sphincter tone, and ability to squeeze the anus.

No comparative studies have proved that BF is superior to a well-established bowel regime, or any other treatment, but in current practice is considered as the next step if maximal laxative therapy within a bowel regime has failed.

We are not aware of the long-term effects on bowel symptoms; it is likely that disease progression might eventually hinder bowel function further. Another consideration is that the effects might not be permanent, and, like in cognitive behavioral treatment on which BF is based, might be reversed if the behavioral changes are not regularly implemented.

In our view, $\mathrm{BF}$ is a package of care, and as such includes titration, under the guidance of a specialist nurse, use of laxatives and constipating agents.

\section{Transanal irrigation (TAI)}

TAI is a mechanical means of obtaining bowel opening. The device consists of a rubber catheter that is inserted in the rectum, with a balloon that is inflated to keep it in place and create a seal. Water is then irrigated, and when the catheter is removed a bowel action is obtained.

The biggest body of evidence mostly comes from trials in patients with a SCI, but it has been proved to be effective in patients with MS. ${ }^{76,77}$ Patients are trained to use the device at home and then guided by the trainer regarding adjustments to the regime.

TAI has certainly made a big impact on the treatment of NBD; in fact, it not only improves symptoms, but also reduces the time spent for toileting. ${ }^{77}$ It has also proved to be a costeffective treatment, reducing need for hospital admissions, physician's visits, and occurrence of urinary tract infections (UTIs). ${ }^{33}$ The latter is particularly relevant for MS patients, in whom UTIs can cause relapse of symptoms.

Compliance with treatment can be an issue, and patient and/or caregiver engagement is essential, in order to implement the necessary adjustments to obtain a successful outcome.

As for BF, the use of constipating and laxative agents can be used to optimize a bowel regime. The introduction of 
a mechanized device might help in improving compliance, by having a better control and record of usage of variables such as the amount of water irrigated and balloon pressure.

Bowel perforation is a rare occurrence, and TAI should be avoided in patients who had previous pelvic surgery. ${ }^{78}$

\section{Neuromodulation}

Sacral neuromodulation (SNM), the stimulation of the S2-S3 nerve root, can be achieved via the sacral nerve or peripherally via the tibial nerve.

Sacral nerve stimulation (SNS) is a two-stage procedure. After a 2-week test period, if a positive effect is recorded via a bowel diary, then a permanent SNS is implanted. This treatment modality has been employed in a small number of patients with an incomplete SCI, and improvement in FI ranging between $59 \%$ and $92 \%$ has been reported..$^{79}$ Retrospectively, a significant improvement in symptoms of FI was noticed in patients who had an SNS implanted for bladder dysfunction. Effects on constipation are less certain.

Use of SNS in patients with MS is only anecdotal, and, learning from the bladder experience, should be reserved to selected patients with stable relapsing remitting disease who had no relapses for at least 2 years ${ }^{80} \mathrm{~A}$ crucial issue is not only the progression of disease and consequent loss of efficacy, but also the need for MRI scans which might require explantation of the stimulator.

Posterior tibial nerve stimulation has shown promising results for the treatment of bladder dysfunction in MS patient ${ }^{80}$ but studies on bowel dysfunction are very much needed.

The use of an anal plug could be considered in the presence of refractory soiling. ${ }^{81}$

\section{Surgery}

Antegrade irrigation with water or osmotic agents, commonly known as the antegrade continence enema (ACE) procedure, can be performed via a surgical appendicostomy ${ }^{82}$ or percutaneously (percutaneous endoscopic colostomy). It is very effective in emptying the bowel, but it has been predominantly used in children with idiopathic constipation or NBD due to myelomeningocele. Very little but positive data exist in adults, and the appendicostomy may stenose with time ${ }^{83}$

This technique could be particularly attractive for patients with very stubborn symptoms of constipation.

Formation of a colostomy or ileostomy can be a very reasonable option for symptoms of FI, as the procedure reduces time spent with bowel care and can provide independence, improving quality of life for patients. In fact, patients who did have a stoma for NBD stated they wished they had it done sooner, but possible complications such as hernias are more common in patients with neurological diseases than neurologically intact patients. ${ }^{84}$ Furthermore, whilst incontinence and independence of bowel management are often greatly enhanced by stoma formation, impairment of colonic transit is not altered by the procedure, so stoma irrigation or laxative use may continue to be required. In this regard, some surgeons prefer performing a laparoscopic loop ileostomy.

\section{Discussion}

NBD is highly prevalent in patients with MS and is certainly multifactorial. Both constipation and FI can affect these patients, and the impact on their life and overall illness is significant. Whilst duration of disease and disability might make it more likely to develop NBD, even patients with short duration and stable disease, with minor disability, might have symptoms. Doctors and nurses dealing with MS patients must be aware of it, and assess NBD in every patient with MS, and effective treatment can improve anxiety and depression and the need of medical interventions.

Constipation may relate to neurological processes that affect ability to identify rectal filling, colonic motility, pelvic dyssynergia, and reduced ability to expel feces due to weakened abdominal wall. Non-neurological factors include medications and lifestyle or behavioral choices. FI is caused by anorectal hyposensitivity, weakness of the anal sphincter, or uncontrolled colonic contractions. Non-MS factors can be represented by diabetes and obstetric injuries, as well as drugs and lifestyle habits. Anorectal hyposensitivity could be considered a common factor to both constipation and incontinence, and justify coexistence of these two symptoms.

Involvement of the spinal cord by the disease appears to have a central role in the pathophysiology of bowel dysfunction, and accumulation of disease might eventually lead to bowel symptoms. These symptoms can occur in patients with preserved mobility and low EDSS, suggesting pin-point involvement of areas of the spinal cord or of alternatively localized disease in the supra-spinal area modulating defecation. However, this is only a speculative thought.

The Cochrane review in 2014 focused on the evidence to support the management strategies for individuals with NBD, and from 20 RCTs only two were exclusively related to NBD in patients with MS. Therefore, the level of evidence was not adequate to be able to make any strong recommendations for the management of NBD in patients with MS. ${ }^{5}$ However, no intervention has absolute contraindication, and a structured approach, employing all the existing armamentarium and the 


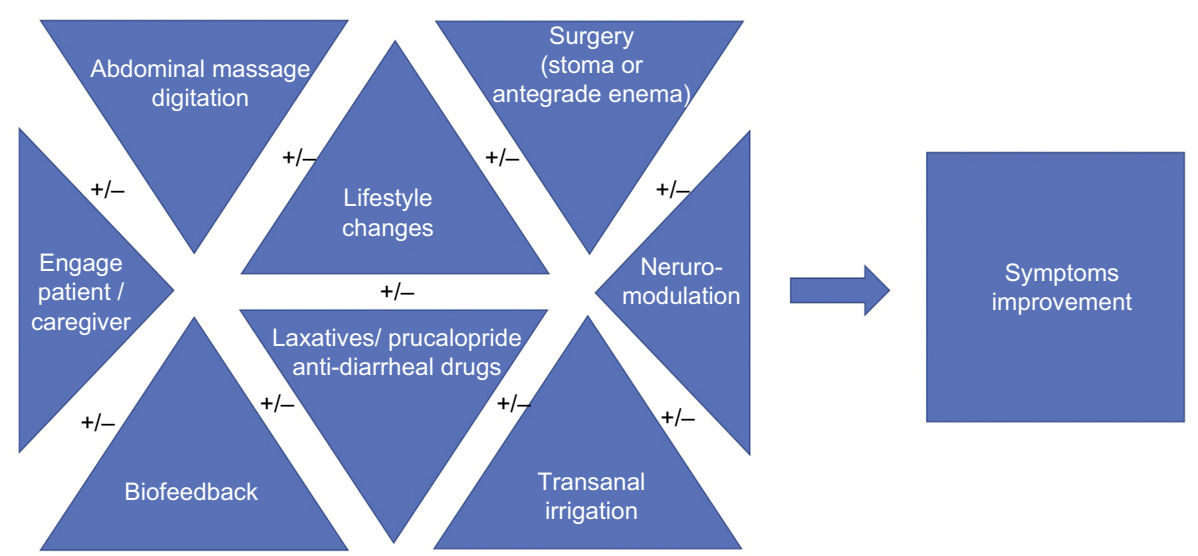

Figure 4 This diagram shows how important it is to tailor treatment, sometimes using a combination of interventions. A stepwise approach is recommended; however, it is paramount to engage patient and caregiver, to understand their preferences and adjust treatment accordingly.

lessons learned particularly with SCI patients, should allow the development of a bowel regime and a routine. ${ }^{6}$

The approach to NBD has been described as a pyramidal stepwise approach, where after failing medical treatment, BF, TAI, and then surgery should be considered in this sequence. But a lack of evidence and clinical experience suggests that a Rubik's cube rather than a pyramid might be a better example of how to approach treatment for NBD.

For example, BF might help enhance the effects of TAI, by maximizing rectal emptying and reducing some of the side effects such as persistent leakage of irrigation fluids. Use of laxatives and/or constipating agents might improve effects of BF or TAI. SNM could enhance the effects of BF, or a patient who had a stoma for FI might benefit from the use of laxatives (Figure 4).

Having said that, a stepwise approach is sensible, and what will make the difference in outcomes, we think, is engaging with the patient and understanding what their preferences might be.

As discussed, treatment needs to be tailored, and require the patient and caregiver to understand that there might not be a "quick fix", and that rather a package of care might be required to be developed over a period of time.

Patient's choice and availability of treatment also play an important role. We have discussed how provision of BF is inconsistent, and, for example, young patients, with combined constipation and incontinence, might find it very convenient to use TAI as a first-line treatment, as TAI will reduce time for toileting and give the confidence of an empty rectum.

At present, neuromodulation has very little scope for the treatment of NBD, but the development of MRI-friendly SNS might open possibility for sizeable studies in patients with
MS-related NBD. Another attractive SNM modality would be transcutaneous nerve stimulation, which would avoid the need for weekly clinical attendance for needle stimulation.

The ACE procedure could be a viable option for stubborn constipation, and formation of a stoma for incontinence should not be a last-ditch option.

\section{Disclosure}

The authors report no conflicts of interest in this work.

\section{References}

1. Browne P, Chandraratna D, Angood C, et al. Atlas of multiple sclerosis 2013: a growing global problem with widespread inequity. Neurology. 2014;83(11):1022-1024.

2. Compston A. Making progress on the natural history of multiple sclerosis. Brain. 2006;129(Pt 3):561-563.

3. Kurtzke JF. Rating neurologic impairment in multiple sclerosis: an expanded disability status scale (EDSS). Neurology. 1983;33(11):1444-1452.

4. Losseff NA, Webb SL, O'Riordan JI, et al. Spinal cord atrophy and disability in multiple sclerosis. A new reproducible and sensitive MRI method with potential to monitor disease progression. Brain. 1996;119(Pt 3):701-708.

5. Coggrave M, Norton C, Cody JD. Management of faecal incontinence and constipation in adults with central neurological diseases. Cochrane Database Syst Rev. 2014;1(1):CD002115.

6. Cotterill N, Madersbacher H, Wyndaele JJ, et al. Neurogenic bowel dysfunction: clinical management recommendations of the Neurologic Incontinence Committee of the Fifth International Consultation on Incontinence 2013. Neurourol Urodyn. 2018;37(1):46-53.

7. Bakke A, Myhr KM, Grønning M, Nyland H. Bladder, bowel and sexual dysfunction in patients with multiple sclerosis--a cohort study. Scand J Urol Nephrol Suppl. 1996;179:61-66.

8. Hinds JP, Eidelman BH, Wald A. Prevalence of bowel dysfunction in multiple sclerosis: a population survey. Gastroenterology. 1990;98(6):1538-1542.

9. Hinds JP, Wald A. Colonic and anorectal dysfunction associated with multiple sclerosis. Am J Gastroenterol. 1989;84(6):587-595.

10. Munteis E, Andreu M, Téllez MJ, Mon D, Ois A, Roquer J. Anorectal dysfunction in multiple sclerosis. Mult Scler. 2006;12(2):215-218. 
11. Nordenbo AM, Andersen JR, Andersen JT. Disturbances of ano-rectal function in multiple sclerosis. J Neurol. 1996;243(6):445-451.

12. Lawthom C, Durdey $P$, Hughes T. Constipation as a presenting symptom. Lancet. 2003;362(9388):958.

13. Drossman DA. The functional gastrointestinal disorders and the Rome III process. Gastroenterology. 2006;130(5):1377-1390.

14. Longstreth GF, Thompson WG, Chey WD, Houghton LA, Mearin F, Spiller RC. Functional bowel disorders. Gastroenterology. 2006;130(5):1480-1491.

15. Whitehead WE, Wald A, Diamant NE, Enck P, Pemberton JH, Rao SS. Functional disorders of the anus and rectum. Gut. 1999;45(Suppl 2):ii55-ii59.

16. Nelson R, Norton N, Cautley E, Furner S. Community-based prevalence of anal incontinence. JAMA. 1995;274(7):559-561.

17. Heaton KW, Radvan J, Cripps H, Mountford RA, Braddon FE, Hughes AO. Defecation frequency and timing, and stool form in the general population: a prospective study. Gut. 1992;33(6):818-824.

18. Khan F, Mcphail T, Brand C, Turner-Stokes L, Kilpatrick T. Multiple sclerosis: disability profile and quality of life in an Australian community cohort. Int $J$ Rehabil Res. 2006;29(2):87-96.

19. Norton C, Chelvanayagam S. Bowel problems and coping strategies in people with multiple sclerosis. Br J Nurs. 2010;19(4):220-226.

20. Bauer HJ, Firnhaber W, Winkler W. Prognostic criteria in multiple sclerosis. Ann N Y Acad Sci. 1965;122:542-551.

21. Dibley L, Coggrave M, Mcclurg D, Woodward S, Norton C. "It's just horrible": a qualitative study of patients' and carers' experiences of bowel dysfunction in multiple sclerosis. J Neurol. 2017;264(7):1354-1361.

22. Preziosi G, Raptis DA, Storrie J, Raeburn A, Fowler CJ, Emmanuel A Bowel biofeedback treatment in patients with multiple sclerosis and bowel symptoms. Dis Colon Rectum. 2011;54(9):1114-1121.

23. Burnett C, Palsson O, Whitehead WE, Drossman D. Psychological distress and impaired quality of life in patients with functional anorectal disorders. Gastroenterology. 1998;114(Suppl 1):A729.

24. Nehra V, Bruce BK, Rath-Harvey DM, Pemberton JH, Camilleri M. Psychological disorders in patients with evacuation disorders and constipation in a tertiary practice. Am J Gastroenterol. 2000;95(7):1755-1758.

25. Correa GI, Rotter KP. Clinical evaluation and management of neurogenic bowel after spinal cord injury. Spinal Cord. 2000;38(5):301-308.

26. de Looze D, van Laere M, de Muynck M, Beke R, Elewaut A. Constipation and other chronic gastrointestinal problems in spinal cord injury patients. Spinal Cord. 1998;36(1):63-66.

27. Han TR, Kim JH, Kwon BS. Chronic gastrointestinal problems and bowel dysfunction in patients with spinal cord injury. Spinal Cord. 1998;36(7):485-490.

28. Harari D, Sarkarati M, Gurwitz JH, Mcglinchey-Berroth G, Minaker KL. Constipation-related symptoms and bowel program concerning individuals with spinal cord injury. Spinal Cord. 1997;35(6):394-401.

29. Lynch AC, Dobbs BR, Keating J, Frizelle FA. The prevalence of faecal incontinence and constipation in a general New Zealand population; a postal survey. $N Z$ Med J. 2001;114(1142):474-477.

30. Menter R, Weitzenkamp D, Cooper D, Bingley J, Charlifue S, Whiteneck G. Bowel management outcomes in individuals with long-term spinal cord injuries. Spinal Cord. 1997;35(9):608-612.

31. Sonnenberg A, Tsou VT, Müller AD. The "institutional colon": a frequent colonic dysmotility in psychiatric and neurologic disease. $\mathrm{Am} J$ Gastroenterol. 1994;89(1):62-66.

32. Thomas S, Mynors G, Simpson S, Meade N, Bowen A. Measuring the burden of hospitalisation in multiple sSclerosis: a cross-sectional analysis of the English Hospital Episode Statistics database 2009-2014. 2015. Available from: http://www.nhis.com/ms-report. Accessed January $1,2018$.

33. Passananti V, Wilton A, Preziosi G, Storrie JB, Emmanuel A. Longterm efficacy and safety of transanal irrigation in multiple sclerosis Neurogastroenterol Motil. 2016;28(9):1349-1355.

34. Emmanuel A, Kumar G, Christensen P, et al. Long-term cost-effectiveness of transanal irrigation in patients with neurogenic bowel dysfunction. PLoS One. 2016;11(8):e0159394.
35. Rapps N, van Oudenhove L, Enck P, Aziz Q. Brain imaging of visceral functions in healthy volunteers and IBS patients. J Psychosom Res. 2008;64(6):599-604.

36. Schweiger M. Method for determining individual contributions of voluntary and involuntary anal sphincters to resting tone. Dis Colon Rectum. 1979;22(6):415-416.

37. Goyal RK, Hirano I. The enteric nervous system. N Engl J Med. 1996; 334(17):1106-1115.

38. Nakayama H, Jørgensen HS, Pedersen PM, Raaschou HO, Olsen TS. Prevalence and risk factors of incontinence after stroke. The Copenhagen Stroke Study. Stroke. 1997;28(1):58-62.

39. Krogh K, Nielsen J, Djurhuus JC, Mosdal C, Sabroe S, Laurberg S. Colorectal function in patients with spinal cord lesions. Dis Colon Rectum. 1997;40(10):1233-1239.

40. Snooks SJ, Swash M. Motor conduction velocity in the human spinal cord: slowed conduction in multiple sclerosis and radiation myelopathy. J Neurol Neurosurg Psychiatry. 1985;48(11):1135-1139.

41. Mathers SE, Ingram DA, Swash M. Electrophysiology of motor pathways for sphincter control in multiple sclerosis. J Neurol Neurosurg Psychiatry. 1990;53(11):955-960.

42. Haldeman S, Glick M, Bhatia NN, Bradley WE, Johnson B, Colonometry JB. Colonometry, cystometry, and evoked potentials in multiple sclerosis. Arch Neurol. 1982;39(11):698-701.

43. Salvioli B, Bharucha AE, Rath-Harvey D, Pemberton JH, Phillips SF. Rectal compliance, capacity, and rectoanal sensation in fecal incontinence. Am J Gastroenterol. 2001;96(7):2158-2168.

44. Scott SM, van den Berg MM, Benninga MA. Rectal sensorimotor dysfunction in constipation. Best Pract Res Clin Gastroenterol. 2011;25(1):103-118.

45. Trivedi PM, Kumar L, Emmanuel AV. Altered colorectal compliance and anorectal physiology in upper and lower motor neuron spinal injury may explain bowel symptom pattern. Am J Gastroenterol. 2016;111(4):552-560.

46. Preziosi G, Raptis DA, Raeburn A, Panicker J, Emmanuel A. Autonomic rectal dysfunction in patients with multiple sclerosis and bowel symptoms is secondary to spinal cord disease. Dis Colon Rectum. 2014;57(4):514-521.

47. Thiruppathy K, Roy A, Preziosi G, Pannicker J, Emmanuel A. Morphological abnormalities of the recto-anal inhibitory reflex reflects symptom pattern in neurogenic bowel. Dig Dis Sci. 2012;57(7): 1908-1914

48. Halligan S, Bartram CI, Park HJ, Kamm MA. Proctographic features of anismus. Radiology. 1995;197(3):679-682.

49. Chia YW, Gill KP, Jameson JS, et al. Paradoxical puborectalis contraction is a feature of constipation in patients with multiple sclerosis. J Neurol Neurosurg Psychiatry. 1996;60(1):31-35.

50. Gill KP, Chia YW, Henry MM, Shorvon PJ. Defecography in multiple sclerosis patients with severe constipation. Radiology. 1994;191(2):553-556.

51. Betts CD, D'Mellow MT, Fowler CJ. Urinary symptoms and the neurological features of bladder dysfunction in multiple sclerosis. $J$ Neurol Neurosurg Psychiatry. 1993;56(3):245-250.

52. Emmanuel AV, Kamm MA. Response to a behavioural treatment, biofeedback, in constipated patients is associated with improved gut transit and autonomic innervation. Gut. 2001;49(2):214-219.

53. Nicoletti R, Mina A, Balzaretti G, Tessera G, Ghezzi A. Intestinal transit studied with radiopaque markers in patients with multiple sclerosis. Radiol Med. 1992;83(4):428-430.

54. Waldron DJ, Horgan PG, Patel FR, Maguire R, Given HF. Multiple sclerosis: assessment of colonic and anorectal function in the presence of faecal incontinence. Int J Colorectal Dis. 1993;8(4):220-224.

55. Glick ME, Meshkinpour H, Haldeman S, Bhatia NN, Bradley WE. Colonic dysfunction in multiple sclerosis. Gastroenterology. 1982;83(5):1002-1007.

56. Porrett T, Cox C. Coping and help-seeking behaviour in women with pelvic floor dysfunction. J Assoc Chart Physiother Women's Health. 2007;101:4-15. 
57. Norton C, Dibley L. Help-seeking for fecal incontinence in people with inflammatory bowel disease. J Wound Ostomy Continence Nurs. 2013;40(6):631E631-638632; quiz.

58. Hazewinkel MH, Sprangers MA, Taminiau-Bloem EF, van der Velden J, Burger MP, Roovers JP. Reasons for not seeking medical help for severe pelvic floor symptoms: a qualitative study in survivors of gynaecological cancer. BJOG. 2010;117(1):39-46.

59. Norton C, Thomas L, Hill J; Guideline Development Group. Management of faecal incontinence in adults: summary of NICE guidance. BMJ. 2007;334(7608):1370-1371.

60. Marrie RA, Reider N, Cohen J, et al. A systematic review of the incidence and prevalence of autoimmune disease in multiple sclerosis. Mult Scler. 2015;21(3):282-293.

61. Preziosi G, Raptis DA, Raeburn A, Thiruppathy K, Panicker J, Emmanuel A. Gut dysfunction in patients with multiple sclerosis and the role of spinal cord involvement in the disease. Eur J Gastroenterol Hepatol. 2013;25(9):1044-1050.

62. Prévinaire JG, Soler JM, Bordji H, Fiolet MC, Navaux MA, Mortier PE. Assessment of severity of neurogenic bowel dysfunction in chronic patients with a simple 1-item questionnaire (PGI-S). Prog Urol. 2016;26(10):e128-581.

63. Wang G, Marrie RA, Fox RJ, et al. Treatment satisfaction and bothersome bladder, bowel, sexual symptoms in multiple sclerosis. Mult Scler Relat Disord. 2018;20:16-21.

64. Emmanuel A. Managing neurogenic bowel dysfunction. Clin Rehabil. 2010;24(6):483-488.

65. Mcclurg D, Hagen S, Hawkins S, Lowe-Strong A. Abdominal massage for the alleviation of constipation symptoms in people with multiple sclerosis: a randomized controlled feasibility study. Mult Scler. 2011;17(2):223-233.

66. Ashraf W, Pfeiffer RF, Park F, Lof J, Quigley EM. Constipation in Parkinson's disease: objective assessment and response to psyllium. Mov Disord. 1997;12(6):946-951.

67. Dahl WJ, Whiting SJ, Isaac TM, Weeks SJ, Arnold CJ. Effects of thickened beverages fortified with inulin on beverage acceptance, gastrointestinal function, and bone resorption in institutionalized adults. Nutrition. 2005;21(3):308-311.

68. Zangaglia R, Martignoni E, Glorioso M, et al. Macrogol for the treatment of constipation in Parkinson's disease. A randomized placebo-controlled study. Mov Disord. 2007;22(9):1239-1244.

69. Nelson $\mathrm{AD}$, Camilleri M, Chirapongsathorn $\mathrm{S}$, et al. Comparison of efficacy of pharmacological treatments for chronic idiopathic constipation: a systematic review and network meta-analysis. Gut. 2017;66(9): 1611-1622.

70. Yi Z, Jie C, Wenyi Z, Bin X, Hongzhu J. Comparison of efficacies of vegetable oil based and polyethylene glycol based bisacodyl suppositories in treating patients with neurogenic bowel dysfunction after spinal cord injury: a meta-analysis. Turk J Gastroenterol. 2014;25(5): $488-492$.
71. Sajid MS, Hebbar M, Baig MK, Li A, Philipose Z. Use of prucalopride for chronic constipation: a systematic review and meta-analysis of published randomized, controlled trials. J Neurogastroenterol Motil. 2016;22(3):412-422.

72. Medaer R, D'Hooghe B, Guillaume D, et al. Efficacy and tolerability of prucalopride in patients with constipation due to multiple sclerosis: a double-blind placebo-controlled pilot study. Gut. 1999;45(Suppl V): A137.

73. Engel BT, Nikoomanesh P, Schuster MM. Operant conditioning of rectosphincteric responses in the treatment of fecal incontinence. $N$ Engl J Med. 1974;290(12):646-649.

74. Munteis E, Andreu M, Martinez-Rodriguez J, Ois A, Bory F, Roquer J. Manometric correlations of anorectal dysfunction and biofeedback outcome in patients with multiple sclerosis. Mult Scler. 2008;14(2):237-242.

75. Wiesel PH, Norton C, Roy AJ, Storrie JB, Bowers J, Kamm MA. Gut focused behavioural treatment (biofeedback) for constipation and faecal incontinence in multiple sclerosis. J Neurol Neurosurg Psychiatry. 2000;69(2):240-243.

76. Preziosi G, Gosling J, Raeburn A, Storrie J, Panicker J, Emmanuel A. Transanal irrigation for bowel symptoms in patients with multiple sclerosis. Dis Colon Rectum. 2012;55(10):1066-1073.

77. Christensen P, Krogh K, Buntzen S, Payandeh F, Laurberg S. Long-term outcome and safety of transanal irrigation for constipation and fecal incontinence. Dis Colon Rectum. 2009;52(2):286-292.

78. Christensen P, Krogh K, Perrouin-Verbe B, et al. Global audit on bowel perforations related to transanal irrigation. Tech Coloproctol. 2016;20(2):109-115.

79. Worsøe J, Rasmussen M, Christensen P, Krogh K. Neurostimulation for neurogenic bowel dysfunction. Gastroenterol Res Pract. 2013;2013:563294-563298.

80. Barboglio Romo PG, Gupta P. Peripheral and sacral neuromodulation in the treatment of neurogenic lower urinary tract dysfunction. Urol Clin North Am. 2017;44(3):453-461.

81. Krassioukov A, Eng JJ, Claxton G, Sakakibara BM, Shum S. Neurogenic bowel management after spinal cord injury: a systematic review of the evidence. Spinal Cord. 2010;48(10):718-733.

82. Malone PS, Ransley PG, Kiely EM. Preliminary report: the antegrade continence enema. Lancet. 1990;336(8725):1217-1218.

83. Patel AS, Saratzis A, Arasaradnam R, Harmston C. Use of antegrade continence enema for the treatment of fecal incontinence and functional constipation in adults: a systematic review. Dis Colon Rectum. 2015;58(10):999-1013.

84. Preziosi G, Emmanuel A. Neurogenic bowel dysfunction: pathophysiology, clinical manifestations and treatment. Expert Rev Gastroenterol Hepatol. 2009;3(4):417-423.

85. Preziosi G. Pathophysiology of Bowel Dysfunction in Multiple Sclerosis and the potential for targeted treatment [Doctoral thesis]. London, UK: University College London; 2014.
Degenerative Neurological and Neuromuscular Disease

\section{Publish your work in this journal}

Degenerative Neurological and Neuromuscular Disease is an international, peer-reviewed, open access journal focusing on research into degenerative neurological and neuromuscular disease, identification of therapeutic targets and the optimal use of preventative and integrated treatment interventions to achieve improved outcomes, enhanced

\section{Dovepress}

survival and quality of life for the patient. The manuscript management system is completely online and includes a very quick and fair peer-review system. Visit http://www.dovepress.com/testimonials.php to read real quotes from published authors. 